\title{
Quarterly Report (Reporting Period January 15, 2003 through April 15, 2003)
}

Rose McCallen, Kambiz Salari, Jason Ortega, LLNL; Fred Browand, Mustapha Hammache, Tsun-Ya Hsu, Diego Arcas, University of Southern California; Anthony Leoard, Philippe Chatelain and Mike Rubel, California Institute of Technology; Chris Roy, Larry DeChant, Bassil Hassan, Sandia National Laboratories; Jim Ross, Dale Satran, Steve Walker, J.T. Heineck NASA Ames Research Center; David Pointer, Tanju Sofu, Argonne National Laboratory

U.S. Department of Energy

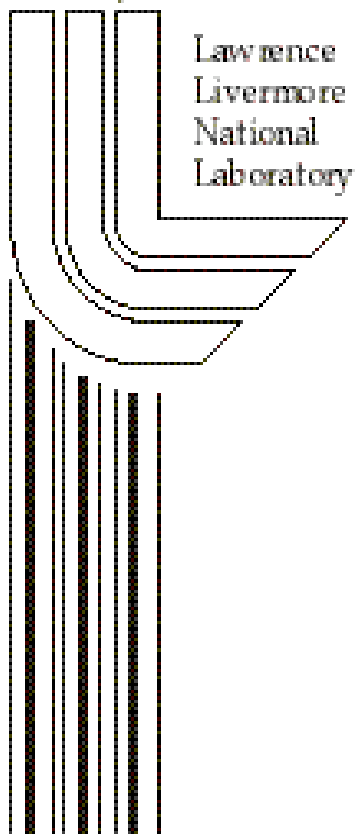

\section{May 15, 2003}




\section{DISCLAIMER}

This document was prepared as an account of work sponsored by an agency of the United States Government. Neither the United States Government nor the University of California nor any of their employees, makes any warranty, express or implied, or assumes any legal liability or responsibility for the accuracy, completeness, or usefulness of any information, apparatus, product, or process disclosed, or represents that its use would not infringe privately owned rights. Reference herein to any specific commercial product, process, or service by trade name, trademark, manufacturer, or otherwise, does not necessarily constitute or imply its endorsement, recommendation, or favoring by the United States Government or the University of California. The views and opinions of authors expressed herein do not necessarily state or reflect those of the United States Government or the University of California, and shall not be used for advertising or product endorsement purposes.

This work was performed under the auspices of the U. S. Department of Energy by the University of California, Lawrence Livermore National Laboratory under Contract No. W-7405-Eng-48.

This report has been reproduced directly from the best available copy.

Available to DOE and DOE contractors from the

Office of Scientific and Technical Information

P.O. Box 62, Oak Ridge, TN 37831

Prices available from (423) 576-8401

http: / / apollo.osti.gov/bridge/

Available to the public from the National Technical Information Service

U.S. Department of Commerce 5285 Port Royal Rd., Springfield, VA 22161 http:/ / www.ntis.gov/

OR

Lawrence Livermore National Laboratory Technical Information Department's Digital Library http:/ / www.llnl.gov/tid/Library.html 


\section{Quarterly Report}

Reporting Period: January 15, through April 15, 2003

Project Title: $\quad$ Aerodynamic Design of Heavy Vehicles

Investigators: $\quad$ Rose McCallen, Kambiz Salari, and Jason Ortega Lawrence Livermore

National Laboratory

Fred Browand, Mustapha Hammache, Tsun-Ya Hsu, Diego Arcas, University

of Southern California

Anthony Leonard, Philippe Chatelain, and Mike Rubel, California Institute

of Technology

Chris Roy, Larry DeChant, and Basil Hassan, Sandia National Laboratories

Jim Ross, Dale Satran, Steve Walker, and J.T. Heineck, NASA Ames

Research Center

David Pointer and Tanju Sofu, Argonne National Laboratory

\subsection{Activities and Accomplishments}

\subsection{Summaries}

Attached are summaries of the activities and accomplishments during this second quarter reporting period for each of the consortium participants. Some of the highlights for this reporting period include:

- Even without the forcing speaker, the "Mozart" device on the GCM geometry provides over $20 \%$ reduction in drag at 0 degree yaw and above $25 \%$ reductions at higher yaw angles

- Experiments and computations guide a conceptual design for reduction of drag due to tractortrailer gap flow

- RANS simulations for the GTS geometry are being finalized for development of clear modeling guidelines with RANS

- Simulations on the GCM geometry have begun

- Vortex methods have improved techniques for the treatment of vorticity near surfaces

\subsection{Statements of Work and Milestones for FY04}

Statements of work with milestones for FY04 were constructed by each organization, reviewed as a group, and discussed over several conference calls with the consortium participants.

\subsection{Collaborations with Industry}

Members of the consortium are responding to two separate requests to participate in industry driven responses to the DOE request for proposals. NASA and LLNL are working with Freightliner in 
constructing one proposal. Another proposal includes industrial participants from Michelin, Freightliner, Swift, and AVL Powertrain with consortium participants from USC, NASA, and LLNL.

\subsection{Publications for United Engineering Conference}

Publisher contracts were finalized for publication of the UEF Conference proceedings. Approximately $70 \%$ of the papers have been received and distributed for peer review. Every effort is being made to collect the remaining papers from conference presenters.

\subsection{Future Plans}

A DOE Aero Team Working Group meeting is planned for May $29^{\text {th }}$ and $30^{\text {th }}$. Industry is invited to participate in the first day of the two day meeting. The second day is reserved for consortium participants to provide a status report, discuss remaining activities for FY03, and review budget plans for FY04. 


\section{Lawrence Livermore National Laboratory}

Activities for this quarter include both computational and experimental efforts. The focus of the computational effort has been to finalize the GTS full vehicle simulations of the NASA Ames 7'x10' wind tunnel experiments and simulations of the gap flow experiments using USC's modified GTS geometry. The data from USC's and NASA's experiments are being used to validate the calculations. The focus of LLNL's discovery experiments conducted in NASA Ames' small-scale 3'x4' wind tunnel is to provide insight into various drag reduction concepts introduced by LLNL. The small tunnel will provide an inexpensive and flexible way to fully investigate the selected drag reduction devices. Our goal is to identify two or more add-on devices targeting the gap, underbody, and the wake of the trailer. The selected add-on devices will then be presented at our next meeting in May to the tractor OEM's in hopes that they will be considered for full-scale testing.

\section{Flow Simulations}

Simulations of the GTS in NASA's 7'x10' wind tunnel and the modified GTS in USC's wind tunnel are complete. Steady and unsteady RANS flow simulations were performed with the OVERFLOW code.

Previous gap flow simulations using a truncated trailer geometry suggests that the trailer wake could significantly impact the unsteady state of the gap flow. To account for this interaction, the complete modified GTS model with the tractor and the full trailer is included in the simulations. URANS simulations are being conducted on the modified GTS geometry with a normalized gap distance of 0.72 which is beyond the critical gap distance of 0.5 . At this gap distance, the flow is unsteady and alternates between symmetric and asymmetric states. The URANS simulation conducted with OVERFLOW clearly indicates an unsteady flow in the gap with periodic interaction with the freestream flow as shown in Figure 1. A new LLNL add-on device that could stabilize the flow in the gap has been tested computationally and the results will be presented at our next meeting in May.

As was mentioned above, we would like to finalize the GTS flow simulations and move on to the GCM geometry. The following simulations have been completed for the GTS in NASA Ames' 7'x10' wind tunnel:

$>0^{\circ}$ yaw, $\mathrm{M}_{\infty}=0.28$, and $\mathrm{Re}=2.0 \times 10^{6}$ based on trailer width

Three turbulence models have been investigated, 1-equation model of Spalart-Allmaras, 2equation models of Wilcox $k-\omega$ and Menter SST. Two-grid resolutions of five and 12 million grid points were used for the grid convergence study.

$10^{\circ}$ yaw, $M_{\infty}=0.28$, and $\mathrm{Re}=2.0 \times 10^{6}$ based on trailer width

Only the 2-equation turbulence models of Wilcox $k-\omega$ and Menter SST are investigated with two grid resolutions similar to the above $0^{\circ}$ yaw case.

\section{Discovery Experiments}

As was mentioned above, the purpose of our experiments in the small-scale NASA Ames 3'x4' wind tunnel is to provide an inexpensive and flexible means of testing and exploring various LLNL drag reduction concepts for heavy vehicles. The construction of the 1/16 scaled wind tunnel model which is similar to the modified GTS and the LLNL add-on devices have been completed. The LLNL discovery 
experiments were started on May 1, 2003 and initial flow visualization about the modified GTS indicates flow separation on the side and the top of the tractor. It is crucial that tractor flow separation be eliminated for these tests since it could adversely impact the evaluation of add-on devices. The flow separations have been successfully eliminated by tripping the flow on the tractor. Performance of various LLNL add-on devices will be presented at our next meeting in May.

\section{Publication}

The report titled, "Ground Transportation System Flow Simulation Using Reynolds-Averaged Navier-Stokes Equations" has been completed and will be published as an LLNL/SNL report. The document is currently undergoing the required review and release process by both organizations.

The trailer wake LES flow simulation results will be published in the UEF conference proceedings. The paper is complete and has been submitted for peer review.

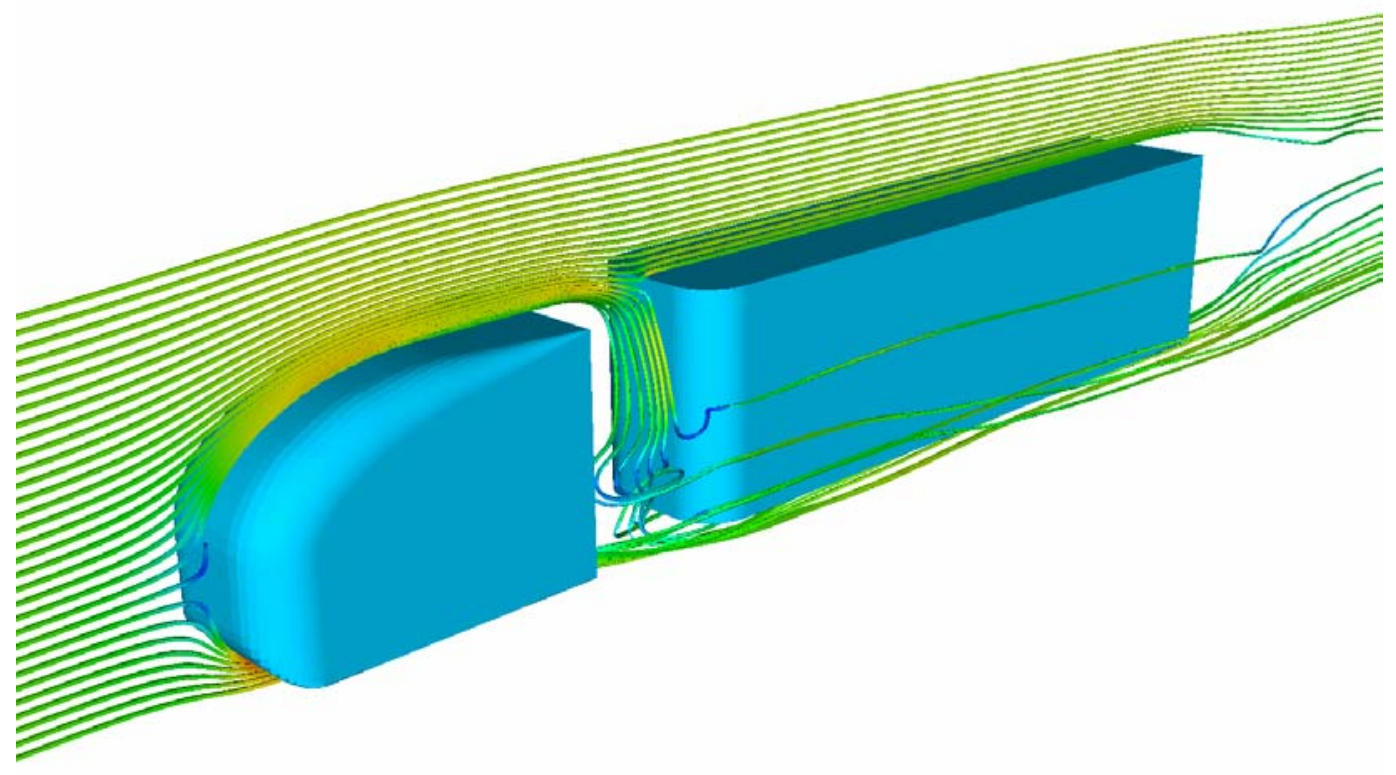

Figure 1. Overflow URANS simulation of modified GTS with a normalized gap distance of 0.72 . Traces are colored by velocity magnitude. 


\section{Sandia National Laboratories}

SNL is responsible for evaluating the use of Reynolds Averaged Navier-Stokes (RANS) to predict the aerodynamic drag on a simplified model of a tractor-trailer vehicle. Over the past few years, Sandia's Navier-Stokes code, SACCARA, has been used to obtain RANS solutions using several different turbulence models. The results of these solutions indicated that the existing meshes did not have small enough spacing normal to the surface of the truck (i.e., $\mathrm{y}+$ in normalized turbulence coordinates). In FY02, one of Sandia's goals was to generate new 3D meshes and to complete the evaluation of the RANS method. A 2D mesh generation study was done to help define required mesh spacing for the 3D meshes.

The finest 3D mesh was completed, and every other grid point was removed to obtain a coarse mesh. The coarse mesh contains about 2.5 million cells and was decomposed, using Sandia's DECOMP code, into 125 zones. The solution was set up to run on 118 processors on the ASCI Red teraflop computer. Some zones were combined on a single processor in order to balance the loading of the processors. Solutions using the Menter hybrid k-omega/k-epsilon and the Wilcox (1998) k-omega turbulence models are fully converged and completed, with preliminary results shown at the United Engineering Foundation conference on the Aerodynamics of Heavy Vehicles.

The fine mesh (20 million cells) was decomposed and run on approximately 950 processors on the ASCI Red machine. Results for the Menter k-omega turbulence model were included in the UEF conference paper submitted in March of 2003. A comparison of the pressure in the base region of the GTS is shown below in Fig. 1 using the Menter k-omega model with both coarse and fine meshes. The differences between the solutions on these two meshes are minor. A comparison of the fine mesh results with experimental data for base pressure is given in Fig. 2. Also shown in this figure are prior results using the Spalart-Allmaras turbulence model that were computed by Kambiz Salari. While the Menter komega results do not match the asymmetric nature of the data found in the experiment, the pressure levels are approximately correct. This is not true for the Spalart-Allmaras model, which significantly underpredicts the pressure near the top and bottom of the base. In order to determine whether the differences between these two computed results are due to the turbulence model or to the grid, the Spalart-Allmaras model is being run on the new fine grid (20 million cells). These results are nearly converged, and will be presented in the final UEF conference paper.

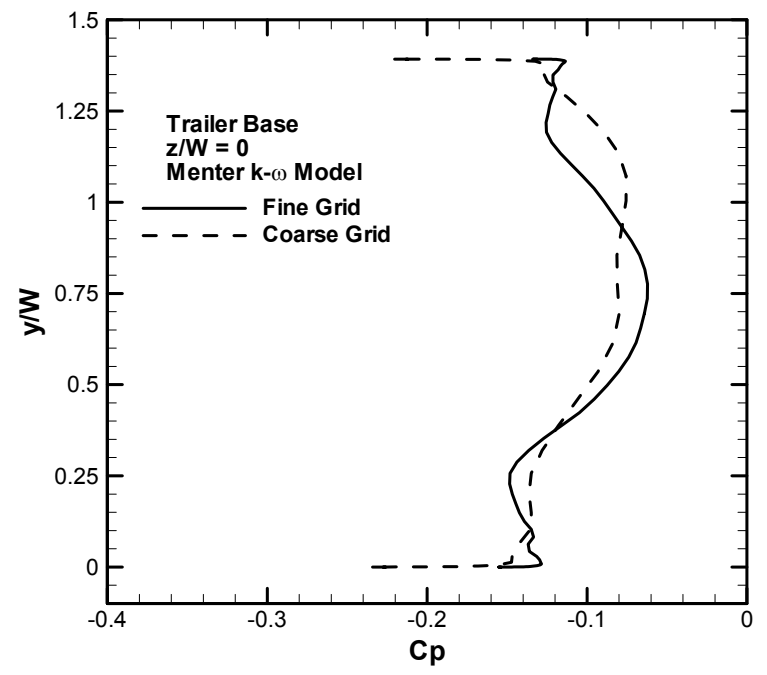

Fig. 1. Base pressure using two grids (Menter model)

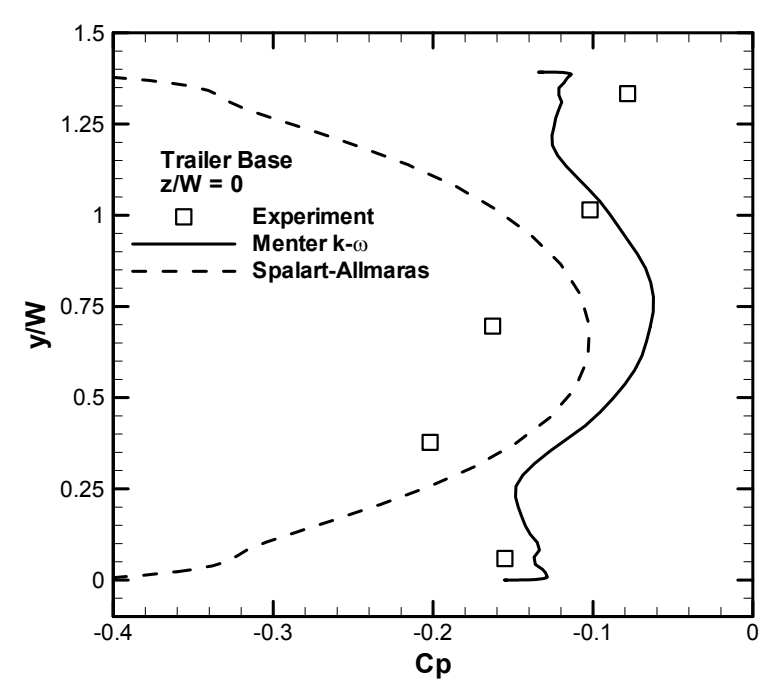

Fig. 2. Base pressure using two turbulence models 


\section{California Institute of Technology}

Caltech's activities for the first quarter included continued research on boundary treatment, high order isotropic redistribution, development of a fast algorithm for the Closest Point Transform and adaptive time integration.

The development of near wall elements is in progress. Our code now includes the new fast algorithm for distance computation and can redistribute particles around an arbitrary geometry.

We pursued the work on the redistribution schemes in Face Centered Cubic (FCC) lattices. A higher order scheme was designed which shows remarkable isotropy when compared to equivalent schemes for cubic lattices (Figs. 1 and 2). Let us emphasize that the redistribution of the vortex particles every few time steps is critical for the convergence of the method, hence the importance of its isotropy and accuracy.

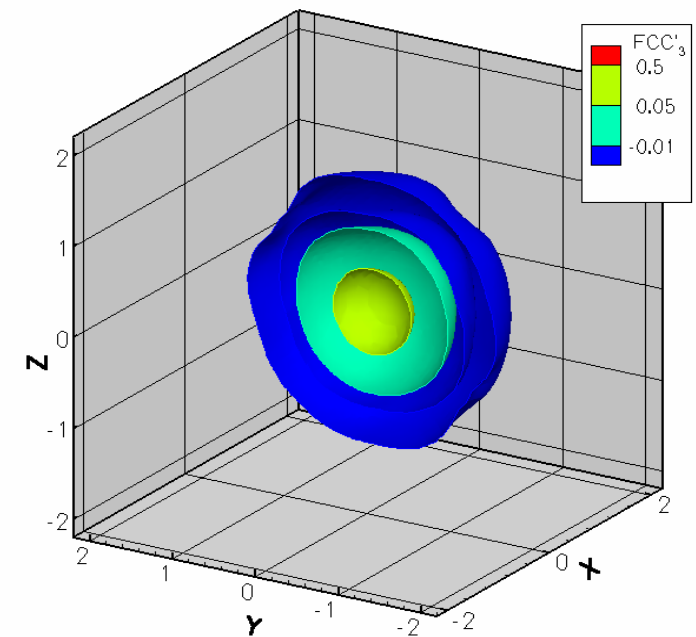

Figure 1: Isosurfaces of the third order FOC scheme $\left(F C C_{3}\right)$

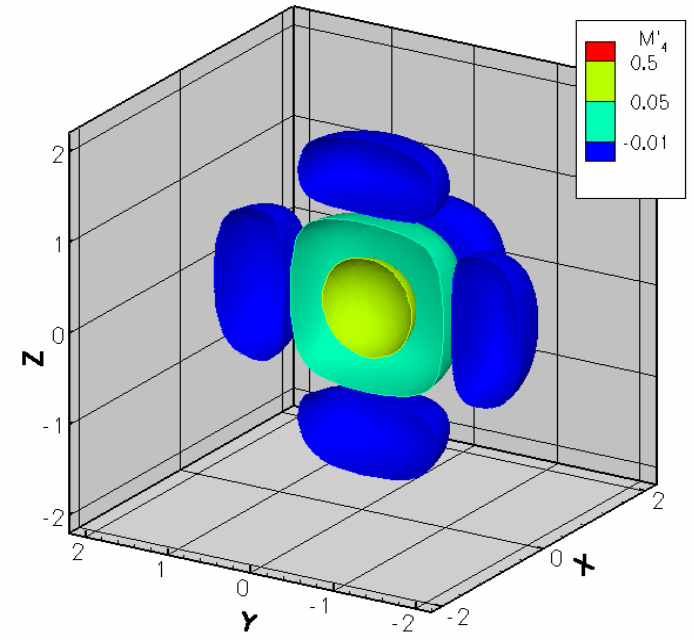

Figure 2: Isosurfares of the third order cubic scheme $\left(M_{4}^{\prime}\right)$

A paper on the new Characteristic / Oct Tree (C/OT) algorithm, which allows the code to compute flow around arbitrary bodies in reasonable time, is currently in preparation. The algorithm works by separating space into families of characteristics of an Eikonal equation and sorting them according to intersection with cells of an oct tree (Figs. 3 and 4).

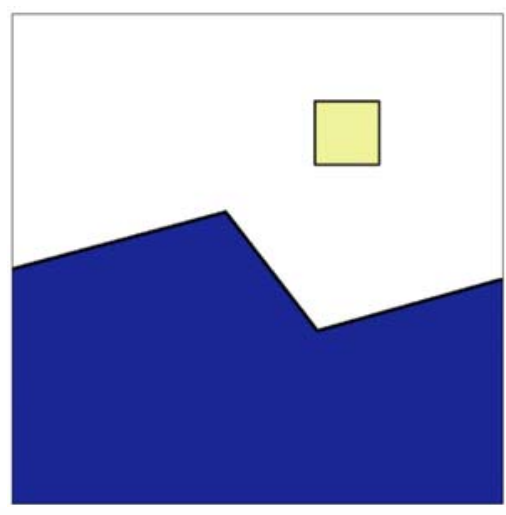

Figure 3: Boundary and oell of interest

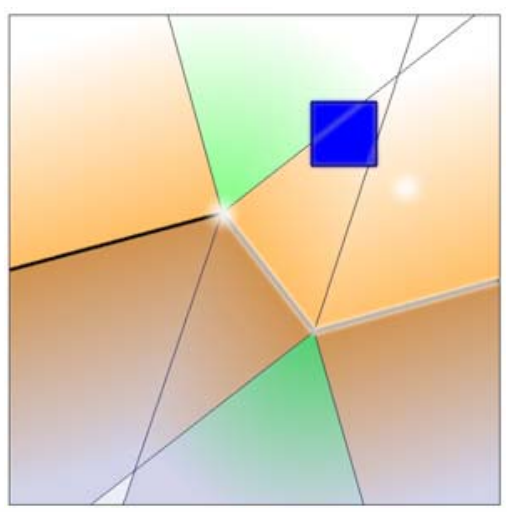

Figure 4: Characteristic regions and intersection with oell 


\section{NASA Ames Research Center}

The 12-ft test of the GCM is still ongoing. Modifications to the PIV system enabled data acquisition in the gap between the tractor and trailer at 1 atmosphere. At 6 atmospheres, the lasers could not be aligned to acquire data. We were able to align the lasers at almost 4 atmospheres. After completing the gap surveys, it was discovered that one of the lasers had been damaged. It has been repaired and further modifications have been added to the PIV system to isolate the lasers from the pressure environment. In late April, the wake surveys will be attempted.
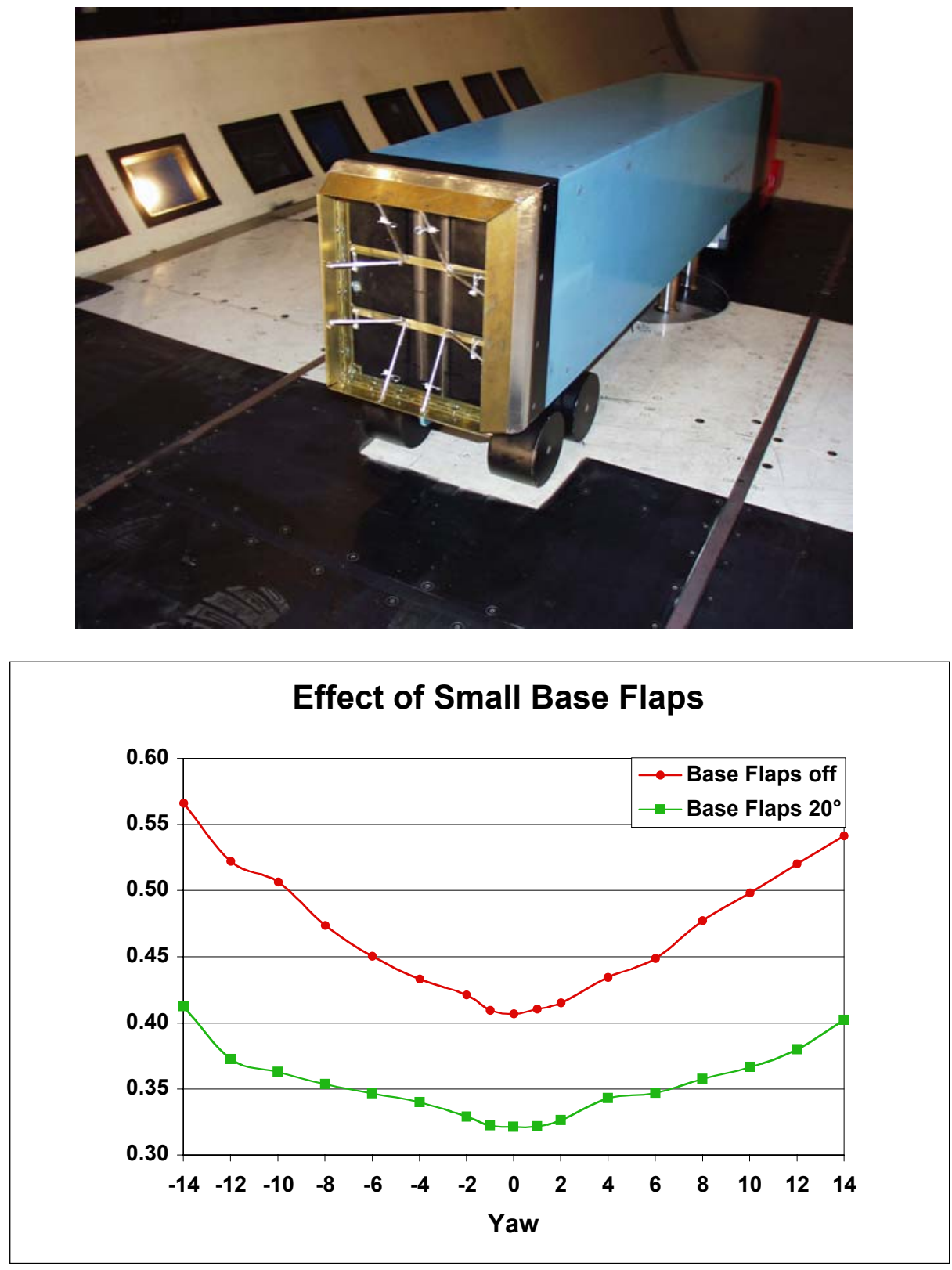

The testing of the small base flaps has shown large drag reduction over the entire yaw range. The base flaps are providing over $20 \%$ drag reduction at zero yaw angle. A plot of drag coefficient versus yaw angle is included with a photo of the base flaps. 


\section{University of Southern California}

\section{Experiments}

Continued efforts on the use of base flaps to reduce drag (with and without acoustic forcing) have been focused on the following areas:

- Documenting the working details of the forcing apparatus. Hot film measurements at the slot and downstream of it were obtained for various duty cycles of the forcing speaker. These hot film measurements were performed outside of the wind tunnel and were used to characterize the jet response to the various forcing functions. The data helps to verify the assumption of zero net momentum flux flow across the slot. Moreover, since the forcing amplitude - an important parameter in the proposed forcing technique - is limited by the current design, full documentation of the forcing response is imperative so that it can be optimized. The measurements show that saw-tooth forcing functions are not appropriate to the enhancement of either the blowing or the suction, due to the damping effect of the AC amplifier. Without the AC amplifier, the saw-tooth forcing functions indeed enhance the suction response. Steps are being taken to replace the speaker by a more powerful forcing mechanism.

- Modifying the flap design. Instead of a motorized system of four independent flaps, the new design will utilize fixed flaps. Eight sets of flaps are considered that will have different angles to the free-stream, ranging from 0 to 28 degrees in increments of 4 degrees. One of the improvements of the new design is that the fixed flaps will be joined at the corners, whereas the independent flaps have gaps between them, which make the flow over the corner regions more complicated. The new design is expected to improve flow control over the base region.

- Preparing to perform DPIV measurements of the flow in the base region for various flap angles with and without forcing. Such measurements will provide a global view of the flow structure and will help fine-tuning the flap angle and forcing scheme.

\section{Publications}

Preparation of manuscripts for the proceedings of the UEF conference on heavy vehicle aerodynamics, namely:

- "On the Aerodynamics of Tractor-Trailers", by M. Hammache and F. Browand

- "Base Flaps and Oscillatory Perturbations to Decrease Base Drag" by T-Y Hsu, M. Hammache and F. Browand

- Preparation for a separate paper that summarizes all experiments conducted to date, excluding the base flaps experiments. The paper (to be authored by MH and FKB) will be submitted to either the Journal of Fluid Mechanics or Physics of Fluids. 


\section{PACCAR CRADA}

\section{Argonne National Laboratory}

With the successes of the Generic Conventional Model evaluations in the previous quarter, significant efforts have been made to initiate the activities planned under the cooperative research and development agreement with PACCAR Technical Center (PTC) to evaluate the capabilities available within commercial software for the prediction of aerodynamic drag for heavy vehicles. The most recent releases of the Star-CD software package offered by CD-Adapco Group and the PowerFLOW software package offered by Exa Corporation have been implemented by ANL to ensure that these studies evaluate the status of current technology. David Pointer of ANL visited the PACCAR Technical Center and the University of Washington's Kirsten Wind Tunnel in March 2003 to assist in the finalization of test plans and coordinate expected computational efforts with the current needs of PTC. PTC's wind tunnel experiments are expected to be delayed until early 2004 because of model development challenges and wind tunnel availability. An extension of demo licenses allowing the use of PowerFLOW has been requested to allow for this shift in the test schedule.

\section{Evaluation of NASA GCM Experiments}

The previous quarter's efforts examined the suitability of the basic k-epsilon models in conjunction with logarithmic wall functions for the prediction of the drag coefficient of the standard truck configuration of the GCM model. Because of the good agreement observed between the computational predictions and the experimental measurements, the plan to evaluate more complex turbulence modeling options was put on hold in order to allow a more thorough investigation of the quality of these results. Suggestions for additional evaluations were collected from DOE team members as well as CFD users at PTC and Fred Ross of CD-Adapco Group.

A preliminary study has been completed to evaluate the effects of the computational resolution of the initial surfaces and the computational mesh on the results. Like a standard mesh sensitivity study, these evaluations separately considers both changes in the mesh structure in the whole flow field and changes in the mesh structure in only the near wall region. However, unlike a standard mesh sensitivity study, the resolution of the initial surface definition is altered to match the resolution of the surrounding mesh. Results show the expected trend where increases in resolution improve predictions, with the effects of improvements in near-wall resolution being much more significant than improvements bulk flow field resolution. A separate mesh sensitivity study using the same initial surface resolution for each computational mesh is currently being completed.

A study has also been completed examining the horizontal and vertical scalability of the Star-CD simulation of the standard configuration of the Generic Conventional Model on ANL's 80-node reserve/reserv1 Linux cluster. The problem scales nearly ideally (i.e. doubling the computational investment halves the computational time) in the horizontal sense, where additional processors are employed to complete the same simulation. The problem does not scale as well in the vertical sense, where higher speed processors are employed to complete the same simulation. Differences in network card and motherboard configurations as well as varying amounts of physical memory probably lead to the non-ideal vertical scaling performance curve.

In addition to the ongoing study of mesh structure sensitivity, current efforts are focused on the evaluation of the effects of the dimension of the domain considered on the simulation results. This study should help address questions regarding the need to model wind tunnel geometries when trying to predict drag coefficients that have been corrected to semi-infinite medium conditions. Following these studies, efforts will be focused on the prediction of drag coefficients for cases with non-zero yaw angles. 